\title{
Corela
}

Cognition, représentation, langage

HS-29 | 2019

Questions et exclamations au prisme de plusieurs approches linguistiques

\section{La question et l'interrogation en contexte : point de vue énonciatif}

Jean-Marie MERLE

\section{(2) OpenEdition}

\section{Journals}

Édition électronique

URL : http://journals.openedition.org/corela/8834

DOI : $10.4000 /$ corela.8834

ISSN : 1638-573X

Éditeur

Cercle linguistique du Centre et de I'Ouest - CerLICO

Référence électronique

Jean-Marie MERLE, «La question et l'interrogation en contexte : point de vue énonciatif », Corela [En ligne], HS-29 | 2019, mis en ligne le 06 septembre 2019, consulté le 28 octobre 2019. URL : http:// journals.openedition.org/corela/8834 ; DOI : 10.4000/corela.8834

Ce document a été généré automatiquement le 28 octobre 2019.

\section{(c) (1) (5)}

Corela - cognition, représentation, langage est mis à disposition selon les termes de la licence Creative Commons Attribution - Pas d'Utilisation Commerciale - Partage dans les Mêmes Conditions 4.0 International. 


\title{
La question et l'interrogation en contexte : point de vue énonciatif
}

\author{
Jean-Marie MERLE
}

L'auteur remercie Marie Loiseau pour ses critiques constructives.

\section{Introduction}

1 Cet article traite de la question et de l'interrogation à partir d'un extrait de Sparkling Cyanide, d'Agatha Christie.

2 On peut définir l'interrogation soit comme un statut illocutoire, soit comme une modalité énonciative, et un énoncé interrogatif comme une prédication munie de cette modalité. Etant donné que la modalité est un phénomène à la fois sémantique - qui apporte une contribution, une détermination, au sens construit d'un énoncé - et pragmatique - d'ajustement à une situation inter-énonciative -, et que la force illocutoire mobilise les deux pour faire aboutir une intention - intention de signifier, mais également intention d'obtenir -, pour tenir compte de cas de figure d'une grande diversité $(\$ 1)$, on applique ici le terme d'interrogation ${ }^{1}$ au phénomène général, et on utilise le terme de question ${ }^{2}$ pour l'interrogation directe, qui en est le prototype.

3 Porteur de modalité et de statut illocutoire, l'énoncé interrogatif est motivé en amont (évaluation de données situationnelles ou contextuelles) et en aval (visée énonciative, ajustement inter-énonciatif).

4 L'interrogation canonique (§ 2) - la question - est l'expression d'une incertitude (§ 2.1), qui se structure de telle sorte que soit placé de façon canonique en position initiale le thème de cette incertitude (\$ 2.2). Autrement dit, il s'agit de façon prototypique d'une prédication de problème de connaissance (\$3), dominée par la modalité épistémique. On examinera la plurimodalité sous-jacente $(\S 4)$, caractéristique récurrente de la modalité énonciative, vérifiée également dans le cas de la question canonique. On analysera le statut illocutoire $(\S 5.1)$ de la question canonique et les phénomènes de 
présupposition et de préconstruction aussi bien dans les questions binaires, questions dites fermées ou totales ( $\$ 5.3$ ), que dans les questions paradigmatiques, dites ouvertes ou partielles, ou questions en wh- (\$5.2). On observera également les réponses (\$6), aux questions binaires ( $\$ 6.1$ ) et aux questions en wh- (\$ 6.2), mais également les réponses qui n'apportent pas l'élucidation demandée ( $\$ 6.3$ ).

Les implications pragmatiques de l'interrogation - et donc l'exploitation de la situation inter-énonciative - varient en fonction de son statut discursif: l'incertitude est d'ordinaire soumise à un co-énonciateur pour élucidation dans les questions directes canoniques, mais elle ne l'est que rarement dans les percontatives (« interrogatives indirectes ») (§ 7), de même que dans les interrogatives indirectes libres (§ 8).

Les autres types rencontrés dans le corpus fourni par ce texte - questions-écho, wh- in situ, tags - (\$9) sont susceptibles de donner à l'énoncé ou à un fragment d'énoncé canonique, le temps d'une situation de co-énonciation, l'incertitude épistémique de base, mais, quelle que soit leur interprétation, quel que soit leur rôle, ils ont en commun d'ouvrir et d'exploiter la fenêtre inter-énonciative caractéristique de la question et de son statut illocutoire, qui n'établit pas seulement une situation de coénonciation : elle sollicite le destinataire (co-énonciateur) pour une prise en compte de l'énoncé interrogatif.

\section{Types d'énoncés interrogatifs représentés dans le corpus}

$7 \quad$ Les différents types d'énoncés interrogatifs représentés dans le texte sont illustrés par les exemples suivants.

Question directe binaire:

1. (1. 21) 'Look here, Iris, did Rosemary ever talk to you much?'

'About what?'

'Afraid?'

uestion directe en wh-:

2. (1. 68) 'What did Sandra Farraday think about it?'

Percontative $^{3}$ (interrogative indirecte):

3. (1. 26) She thought she saw what was in his mind.

Question indirecte libre:

4. (1. 2) When had that begun?

Structure déclarative affectée d'un statut illocutoire interrogatif :

5. (1.52-53) 'There wasn't anyone - that you knew of - who - who might have had it in for her?'

Segments interrogatifs indexés sur un énoncé en amont :

6. (1. 68-69) George said: 'What did Sandra Farraday think about it?"

7. (1. 48-49) ‘Did Rosemary ever say she was afraid of anybody?'

8. (1. 50-51) 'What I'm trying to get at is, did Rosemary have any enemies?'

'Amongst other women?'

9. (1. 84) 'Knocked about a good deal, hasn't he?' 


\section{Question canonique - problème de connaissance et thématisation}

\subsection{Problème de connaissance} have any enemies?'), did est en position initiale : la question a pour thème la validation ou la non-validation de la relation prédicative, et c'est le nœud prédicationnel qui est placé en position thématique. Did remplit la fonction nodale, mais est également une contribution à la modalité ${ }^{10}$.

Dans l'exemple 2 ('What did Sandra Farraday think about it ?'), le thème de l'incertitude est représenté par what - placé en position initiale, pronom renvoyant à un paradigme ouvert, sans présélection ${ }^{11}$. Structurellement ${ }^{12}$, c'est la case de la complémentation de think qui est instanciée ${ }^{13}$, et cette instanciation permet d'interpréter le thème de l'interrogation: il s'agit de l'opinion de Sandra Farraday sur le thème auquel fait référence about it. Wh- exprime une non spécification référentielle, à l'intérieur du 
paradigme représenté par what, dans la structure de think (paradigme des contenus de pensée). La question est une sollicitation, une invitation à réinstancier.

Dans l'énoncé 11 ('How intimate was she with them ?'), le thème de l'incertitude est représenté par le syntagme how intimate, l'adverbe how renvoyant au paradigme de la manière. Comme l'anglais n'a pas d'adverbe paradigmatique spécialisé dans la quantification, c'est la combinaison d'une notion gradable (intimate) et de how qui permet de représenter le degré comme problème de connaissance ${ }^{14}$.

Comme dans les questions binaires, l'inversion auxiliaire-sujet (did Sandra Farraday / was she) est structurellement nécessaire dans les questions en wh-pour que l'énoncé s'interprète comme question (directe, directe libre, ou indirecte libre): les subordonnées de plusieurs types - les concessives en wh-, les intégratives nominales (relatives nominales, sans antécédent), les intégratives adverbiales (subordonnées adverbiales ou circonstancielles), les percontatives (interrogatives indirectes) -, qui toutes sont sans inversion, peuvent en effet être antéposées.

\section{L'interrogation canonique, expression d'un problème de connaissance}

La fonction de ces énoncés - la représentation d'une incertitude - montre :

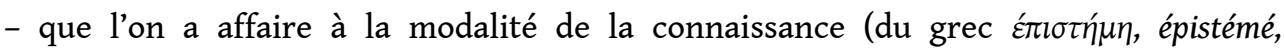
connaissance, savoir), qui concerne à la fois le certain et le non-certain ;

- que la modalité interrogative canonique (incertitude et absence de prise en charge : did Rosemary have any enemies? / What did she think about it ?) est complémentaire de la modalité assertive (certitude et prise en charge : [manipulation] Rosemary had enemies / She thought that $X Y Z)^{15}$;

31 - que lorsqu'un énoncé interrogatif représente un problème de connaissance ${ }^{16}$ - cas particulier mais prototypique -, il relève de la modalité épistémique (did Rosemary have any enemies? 国 perhaps she had enemies / she might have had enemies, etc. / She thought something about it but I don't know what it was).

\subsection{Quatre remarques sur la modalité de la connaissance - assertion (modalité de type 1) et modalité épistémique (modalité de type 2)}

32 1/ La modalité de la connaissance comprend à la fois la modalité de l'assertion (modalité du certain et de la prise en charge énonciative : Rosemary had enemies [ => I know she did : p]) et la modalité épistémique (modalité du non-certain et de l'absence de prise en charge énonciative : perhaps she had enemies / did she have any enemies? [=> I don't know if she did or not: $\mathrm{p}, \mathrm{p}$ ']).

2/ Assertion (modalité de type 1) et modalité épistémique (modalité de type 2) sont complémentaires et s'organisent comme un domaine dans lequel le certain (assertable p) correspond à un intérieur I et le non-certain (non-assertable p,p') à un extérieur E. Cette complémentarité justifie l'adjacence de ces deux modalités ${ }^{17}$. 


\section{énoncé - et un phénomène pragmatique - l'ajustement de l'énoncé à des données situationnelles et à un destinataire, via un processus d'évaluation: elle se décrit} également comme un jugement.

\section{1. (1. 21) 'Look here, Iris, did Rosemary ever talk to you much?'}

Le terme de jugement ne signifie pas que l'énonciateur juge son propre énoncé fini, did Rosemary ever talk to you much. D'où lui viendrait cet énoncé ? Il ne signifie pas non plus qu'il se contente d'ajouter une détermination qui serait interprétable comme jugement, à la relation <Rosemary-talk to you much>. D'où lui viendrait cette relation prédicative ? Son jugement, en amont, fait d'abord émerger des données situationnelles les termes d'une relation pertinente (sélection d'un schéma de lexis pertinent), et justifie l'exploitation de la question, en aval.

40 D'une part, le jugement énonciatif commence donc en amont de l'énonciation. D'autre part, cause et but, d'une manière générale, sont deux composantes de la motivation : la motivation de l'énoncé et de sa détermination modale englobe et sa cause (en amont) et son but (en aval).

41 La question directe de la ligne 21 (ex. 1) est la première question directe d'une série de questions canoniques binaires (1.21, 43, 48, 50, 60, 62, 81, 84-85) et de questions en wh(l. 41, 68, 76, 89) dont la source énonciative est le personnage de George, et le destinataire le personnage d'Iris; et beaucoup plus rarement l'inverse (1.44-45). L'épouse de George, Rosemary, sœur d'Iris, est morte empoisonnée, un an auparavant, le 
jour de son anniversaire (cf. l. 104-110). L'enquête a conclu au suicide, mais George a reçu neuf mois plus tard deux lettres anonymes ('You think your wife committed suicide. She didn't. She was killed.') lui affirmant qu'elle a été assassinée.

Ce qui motive en amont la première question de George, c'est :

1a/ l'idée que la conclusion de l'enquête est erronée; d'où le besoin de rouvrir l'enquête, autrement dit d'en savoir plus (appréciation sous-jacente d'un déficit de connaissance et nécessité d'une remédiation);

$1 b /$ l'idée que certains faits concernant Rosemary, son épouse, lui ont échappé ; d'où le besoin de trouver une source d'information; la sœur de Rosemary peut être cette source (hypothèse sous-jacente d'une source de remédiation);

1c/ l'idée qu'Iris peut en savoir davantage que lui sur sa sœur. La quantification (much) des confidences de Rosemary à sa sœur Iris est en quelque sorte son premier problème de connaissance (hypothèse sous-jacente de résolution du problème).

Pour cette raison, la question portera non sur la validation / non validation de $<$ Rosemary-talk to you > (Rosemary et Iris sont sœurs et vivaient sous le même toit avant la mort de Rosemary), mais sur celle de <Rosemary-talk to you much>, autrement dit sur l'abondance de ses confidences ( $m u$ ch).

Ce qui motive en aval la question de George, c'est :

2a/ l'intention de signifier ce problème de connaissance, did Rosemary ever talk to you much; le thème de la question est double : validation / non validation et quantification de la relation <Rosemary-talk to you $>$ (prise en compte d'un destinataire et quantification de la remédiation à en attendre) ;

$2 \mathrm{~b} /$ l'intention de soumettre ce problème de connaissance à Iris (nécessité de mettre en place une situation inter-énonciative) ;

2c/ l'intention d'obtenir une réponse non seulement à cette question, mais à une cascade de questions (l'intention est de sonder le destinataire puisque les faits à découvrir sont inconnus : une seule question a peu de chance de formuler d'emblée l'inconnue-clé).

1 La question canonique en soi est donc envisageable comme une chaîne modale incluant :

1/ l'évaluation d'une situation (mort de Rosemary à réenvisager comme meurtre) ;

2/ l'évaluation d'un manque à combler (méconnaissance des faits; nécessité de remédiation);

3/ une conjecture concernant l'intimité des deux sœurs ;

4/ une conjecture concernant Iris, envisagée comme source de révélations sur Rosemary ;

5/ nécessité de transmettre et de soumettre le problème de connaissance à un destinataire ; d'où l'ouverture d'une fenêtre inter-énonciative ;

6/ sollicitation du co-énonciateur Iris.

Dans cet enchaînement, on retrouve la plurimodalité intrinsèque à toute modalité énonciative ${ }^{19}$ : ici, appréciation de données situationnelles; émergence d'un problème de connaissance; nécessité de remédiation; conjectures (incertitude); nécessité de soumettre le problème pour élucidation. La plurimodalité sous-jacente à la question, en l'occurrence, est une chaîne d'évaluation, de nécessité, de conjecture. 
59 C'est de cette chaîne que vient le caractère présupposant de la question, qu'elle soit question en wh- (\$ 5.2) ou question binaire (\$ 5.3).

L'aboutissement de cette chaîne est la sollicitation inter-énonciative: la question prototypique énonce un problème de connaissance et le transmet au co-énonciateur pour résolution, mais également, de façon plus large, pour sonder le co-énonciateur.

On peut faire l'hypothèse que la sollicitation inter-énonciative est une caractéristique invariante de la question, qu'elle soit l'expression d'un problème de connaissance ou question rhétorique.

\section{Question directe : statut illocutoire, présupposition, préconstruction}

\subsection{Statut illocutoire}

1. (1. 21) 'Look here, Iris, did Rosemary ever talk to you much?'

Cet exemple, première question d'une série de questions que se remémore Iris, montre bien les mécanismes inter-énonciatifs propres à la question directe.

Look here, Iris inaugure une situation de co-énonciation et opère une focalisation. L'apostrophe Iris donne à Iris le statut de co-énonciateur. Look here a les caractéristiques de l'injonction (Look est un impératif), qui active la situation inter-énonciative (look), et matérialise la sollicitation du co-énonciateur tout en opérant un repérage déictique (here), par indexation sur la situation d'énonciation. L'ensemble de ces opérations énonciatives (apostrophe, injonction, indexation) sert à la fois à mettre en place une situation de co-énonciation (discours direct dans une situation d'énonciation dérivée ${ }^{20}$ ), à identifier le destinataire, à lui faire connaître son statut de co-énonciateur, et à focaliser sur l'inconnue qui motive l'énoncé, did Rosemary ever talk to you much[ ?]. Le point d'interrogation représente graphiquement l'ouverture d'une fenêtre interénonciative, suggère un contour intonatif particulier (cf. l'article de Stephan Wilhelm dans le présent volume), même si lors de la lecture l'intonation reste muette, mentale, et opère l'indexation du contenu énonciatif de la question sur le destinataire. C'est l'aboutissement de toute une chaîne, comme on l'a vu au § 4, par laquelle l'énonciateur conçoit un problème de connaissance, la nécessité d'y remédier, et soumet ce problème au co-énonciateur pour résolution.

L'apostrophe a pour fonction, comme on vient de le voir, d'instaurer un co-énonciateur (ou de conférer à un destinataire le rôle pragmatique d'allocutaire ${ }^{21}$ ) et d'inaugurer une situation de co-énonciation. Chez d'autres auteurs, cette phase inaugurale et l'ouverture de la fenêtre inter-énonciative (la mise en place de la situation interénonciative) pourraient se représenter à l'aide d'une apostrophe et d'une ponctuation interrogative : Iris?

\subsection{Présupposition et préconstruction - Questions en wh-}

La présupposition, dans les questions en wh-, est immédiatement accessible : ce sont des questions partielles et l'inconnue ne concerne que l'une des places de la structure, dans sa relation à la structure. Ainsi, dans les questions suivantes, on reconstruit les présupposés : 
66 paradigme du degré associé à la propriété be intimate with them que porte la question (thème de la question donné par l'adverbe how) ;

67 - you mean some sort of confidence (12); la question porte uniquement sur la détermination de sort of confidence (thème de la question donné par le déterminant what)

68 - Sandra Farraday thought something about it (2); la question porte comme on l'a vu au $\$$ 2.2 sur le contenu de pensée de Sandra Farraday, représenté par le pronom what, interprétable par sa fonction de complément de think;

11. (1. 41) 'How intimate was she with them?'

12. (1. 44-45) 'What sort of confidence do you mean?'

2. (1. 68) George said: 'What did Sandra Farraday think about it?'

69 Les questions en wh- sont systématiquement présupposantes, mais les préconstruits contextuels, en amont, et la nature de la source énonciative enrichissent la motivation,

70 - A la ligne 41 (ex. 11), la question est motivée par la réponse de la l. 40 (Gloria King, Mrs Atwell - Maisie Atwell. Jean Raymond), donnée à la question indirecte (percontative enchâssée dans la structure de asked) des 1. 37-38 : who Rosemary's best women friends had been. Le présupposé est impliqué par la relation Rosemary's best women friends: l'amitié présuppose un certain degré d'intimité. Tout porte à penser que la motivation en aval (l'objectif) est aussi de trouver une nouvelle source d'information éventuelle dans ces trois amies.

71 - Aux lignes 44-45 (ex. 12), la question est posée par Iris à George, et sa motivation directe en amont est la question de George (1.43) do you think she might have confided in any of them[?]. En aval, l'intention de signifier est que l'énonciateur (Iris) attend une spécification (déterminant what thématisé) de la part du co-énonciateur (George), mais l'intention est surtout de le sonder (de le faire parler) pour cerner la nature de ses soupçons.

72 - A la ligne 68 (ex. 2), la question, dont George est la source énonciative, est motivée en amont par le thème des lignes 63-67 (l'intérêt de Rosemary pour la politique), mais plus particulièrement par les énoncés des deux lignes précédentes (1.66-67) dont Iris est la source énonciative (I think Stephen Farraday interested her in them [ = politics]. He used to lend her pamphlets and things.): cf. think about it. En aval, elle est motivée par l'objectif de faire surgir des indices nouveaux.

73 Chacune de ces questions est motivée en amont et en aval selon le modèle donné au $\S 4$, à propos de l'ex. 1, 1. 21 : la motivation varie en fonction du contexte (préconstruit) et de la source énonciative.

\subsection{Présupposition et préconstruction - Questions binaires}

74 Pour les raisons exposées au § 4.4, les questions binaires sont tout autant motivées en amont qu'en aval.

Dans l'exemple 13 (1. 43), le frayage en amont a été opéré en plusieurs étapes : les amies de Rosemary ont été énumérées par Iris (1. 40), ce qui présuppose un degré d'intimité (l. 41, cf. ex 11). La réponse évasive d'Iris (I don't know exactly, 1. 41) motive et entraîne ici une reformulation (rôle de I mean) qui permet de matérialiser précisément la conjecture she might have confided in any of them qui motive la question: George

Corela, HS-29 | 2019 
enchâsse sa conjecture pour la soumettre telle quelle à Iris (do you think $+\mathrm{C}$ ). Autrement dit, il enchâsse sa conjecture dans une conjecture présupposante: you may think she might have confided in one or more of them. Cet enchâssement permet de maintenir le modal might, d'interprétation épistémique, et d'indexer, pour résolution, les deux conjectures sur le co-énonciateur Iris.

Dans l'exemple 14 (1. 48), les réponses demandées à Iris ne sont toujours pas venues. Iris demande une spécification à George (cf. 1. 44-45, ex. 12, §5.2). Comme la motivation initiale ( $\$ 4$, soupçon d'assassinat) demeure pertinente, la question matérialise de la même façon deux présuppositions qui correspondent l'une et l'autre à deux conjectures pertinentes : she may have been afraid of somebody and she may have said so. réponse mais a provoqué une réaction interrogative (1. 49, 'Afraid ?'). Elle entre dans le focus d'une clivée en what (what I'm trying to get at is focus did Rosemary have any enemies?). Il ne s'agit pas d'une reformulation mais d'une remontée dans la chaîne logique de la présupposition: if Rosemary had any enemies (conjecture sous-jacente, 1. 50), she might have known, and she might have been afraid (conjecture sous-jacente, 1. 48).

13. (1. 43) 'I mean, do you think she might have confided in any of them?'

14. (1. 48) 'Did Rosemary ever say she was afraid of anybody?'

10. (1.50) 'What I'm trying to get at is, did Rosemary have any enemies?'

Toutes ces questions sont soumises par George à Iris. Dans toutes ces questions, c'est le nœud prédicationnel qui est thématisé $(13:$ do $; 10,14:$ did $)$ et chacune de ces questions est donc binaire. Chacun de ces énoncés est un problème de connaissance et relève in fine de la modalité épistémique. La fenêtre inter-énonciative est explicitement ouverte (rôle de la question directe). Chacun de ces problèmes de connaissance est soumis au co-énonciateur (Iris) pour élucidation. Par-delà l'intention de signifier ce problème de connaissance, la motivation en aval est d'obtenir des données nouvelles, inconnues jusqu'à présent.

Les questions binaires, comme les questions en wh-, sont motivées en amont (évaluation des données, identification d'un problème de connaissance, nécessité d'une remédiation, conjecture pertinente) comme en aval (intention de signifier et intention d'obtenir une élucidation large dans le cas de la question canonique). Le choix d'un schéma de lexis (notions lexicales à structurer) et d'une structuration (relation prédicative) est en accord avec les données préconstruites. La relation interénonciative est activée pour solliciter le destinataire et lui soumettre le contenu propositionnel (prédication, ou relation énonciative).

\section{Réponses}

80 Le problème de connaissance (modalité épistémique) est indexé sur l'énonciateur dans un premier temps, et la fonction pragmatique et inter-énonciative de la question directe est de le ré-indexer sur le co-énonciateur, qui est sollicité pour le résoudre. Dans les questions binaires, la réponse attendue est l'assignation d'une polarité au contenu propositionnel en question. Dans le cas des questions en wh-, la réponse se fait par réinstanciation (\$ 6.2). Mais il est toujours possible de se soustraire à la sollicitation (§ 6.3) : le problème de connaissance n’est alors pas élucidé. 


\subsection{Réponses aux questions binaires}

81 Les questions binaires appellent une réponse en yes / no, qui assigne au contenu propositionnel en question une polarité, comme dans l'extrait 15 ou dans l'extrait 16 :

15. (1. 60-61) 'Did she ever talk about them?'

'No, I don't think so.'

16. (1.94-98) 'Rosemary saw rather a lot of Anthony Browne, didn't she?'

'Yes - yes, she did.'

82 En 15, la polarité de la réponse est négative ( $\mathrm{No}$ ), et p' est sélectionné, mais le second segment (I don't think so) introduit un élément de subjectivité (I don't think + Adv), et ipso facto réintroduit une incertitude relative. Dans l'extrait 16, la polarité choisie est yes, et $\mathrm{p}$ est sélectionné, mais le tiret et la réitération de yes marquent également une hésitation, hésitation non sur la polarité en elle-même, mais sur la motivation de l'énoncé 'Rosemary saw rather a lot of Anthony Browne, didn't she?' (les deux personnages s'interrogent l'un l'autre et l'un sur l'autre, mais Iris hésite à révéler ses certitudes et ses soupçons).

\subsection{Questions en wh- et réinstanciation}

83 L'extrait 17 (1. 13-14), ci-dessous, montre que la réponse n'est qu'une réinstanciation: l'énoncé n'a pas à être restructuré intégralement (Nothing's the matter); la réponse ne fournit (canoniquement) que la référence destinée à réinstancier la case pertinente (ouverte par what, 1. 14). L'indéfini nothing est un pronom paradigmatique, tout comme le pronom what, et sa détermination spécifie que la ré-instanciation est négative: aucun élément du paradigme n'est pertinent.

17. (l. 13-14) [...] to her question as to what was the matter, he replied briefly, ' Nothing.'

18. (1.37-40) Another day he asked her suddenly who Rosemary's best women friends had been.

Iris reflected.

'Gloria King. Mrs Atwell - Maisie Atwell. Jean Raymond.'

Dans l'extrait 18, la réponse se fait en extension (énumération de tous les éléments du paradigme) et, comme dans l'extrait précédent, la ré-instanciation s'opère sans restructuration complète : Gloria King, Mrs Atwell - Maisie Atwell. Jean Raymond (1. 40), et non Rosemary's best women friends were Gloria King, Mrs Atwell - Maisie Atwell, Jean Raymond).

La ré-instanciation est nécessairement indexée sur (repérée par rapport à) l'énoncé qui précède, ce qui suffit donc pour en faire un énoncé viable.

On a ainsi affaire à un frayage (référentiel et discursif) qui s'appuie sur l'instanciation opérée à l'aide de wh-. La réponse est une ré-instanciation.

\subsection{Réponses sans élucidation}

On remarque dans ce texte que la modalité épistémique est maintenue le plus souvent de diverses façons dans les réponses.

19. (1. 57-59) How much had Rosemary seen of the Farradays?

'I really don't know.'

20. (1. 89-90) 'What's his business?' 
'I don't know.'

21. (1. 43-44) '[...] do you think she might have confided in any of them?'

'I don't really know. I don't think it's awfully likely... What sort of confidence

do you mean?' connaissance représenté par what was in his mind, dont la solution est entrevue (realized / could guess pourraient commuter avec saw) mais n'est pas donnée dans cet énoncé : une partie de la solution est livrée à droite, mais dans un énoncé différent. 
98 En elle-même, la subordonnée nominale ne suffit pas à identifier un problème de connaissance. Les indices se trouvent en dehors d'elle-même, fournis par le segment introducteur dans lequel elle est enchâssée $($ she saw $+C)$. Une caractéristique de l'interrogative indirecte observée ici (extrait 3,1.26) est que le sujet de l'enchâssante est bien siège d'un problème de connaissance, mais que ce problème de connaissance n'est pas livré à un co-énonciateur à la faveur d'une situation inter-énonciative.

Il y a donc trois différences majeures entre les «interrogatives indirectes » et les questions directes :

1 / toutes les questions directes ne sont pas l'énoncé d'un problème de connaissance (cf. les questions rhétoriques), alors que le contenu propositionnel de toute interrogative indirecte est un problème de connaissance, identifiable comme tel en dehors de luimême, dans la structure enchâssante ;

2/ toutes les questions directes (canoniques ou rhétoriques) mettent en place une situation inter-énonciative servant à solliciter le co-énonciateur, alors que les interrogatives indirectes en elles-mêmes ne sollicitent pas le co-énonciateur (cf. extrait $3,1.26)$, et seul le segment introducteur enchâssant est à même de représenter une sollicitation inter-énonciative, comme dans les extraits 22 et 23 ci-dessous, dans lesquels he asked her $+C$ et her question as to $C$ représentent explicitement une situation inter-énonciative :

22. (1.37) Another day he asked her suddenly who Rosemary's best friends had

been.

23. (1. 13-14) He behaved like a man who has had a shock, but to her question as to

what was the matter, he replied briefly, 'Nothing.'

3/ En tant qu'enchâssées, les « interrogatives indirectes ", à la différence des questions directes, ne sont pas des prédications, ne sont pas porteuses de modalité énonciative et ne peuvent avoir la fonction illocutoire des questions directes ${ }^{24}$. Elles sont dominées par la modalité épistémique via leur dépendance syntaxique, sémantique et énonciative, et c'est leur indexation par enchâssement qui leur donne leur statut « interrogatif ».

\subsection{Les percontatives (« interrogatives indirectes »), et comment les reconnaître}

104 C'est pour cette raison qu'un terme différent se justifie: les subordonnées interrogatives ne disent pas, à la différence des questions directes canoniques - et il s'agit d'une différence majeure -, que le problème de connaissance est soumis à un coénonciateur, ni, d'une manière plus générale, que le co-énonciateur est sollicité (cf. $§ 4$, $\S 5.1$, 7.1, note 16). Elles ne correspondent plus au schéma syntaxique, pragmatique, énonciatif de la question. D'où l'emploi du terme de percontative $e^{25}$.

3. (1. 26) She thought she saw what was in his mind

10. (l. 50) 'What I'm trying to get at is, did Rosemary have any enemies?'

'identification des percontatives (« interrogatives indirectes ») ne fait pas l'unanimité ${ }^{26}$ et elle mérite qu'on s'y arrête.

106 Le problème de connaissance (modalité épistémique) est central. C'est lui qui permet de distinguer les percontatives des intégratives, que rien ne distingue formellement par 
ailleurs (cf. l'intégrative What I'm trying to get at dans What I'm trying to get at is, did Rosemary have any enemies? 1. 50, extrait 10).

On observe que la fonction de l'intégrative (relative nominale, comme what I'm trying to get at, 1. 26, ex. 10 / subordonnée adverbiale, comme when she came, 1.12) varie en fonction du subordonnant en wh- :

108 - 1.26, le subordonnant est le pronom what, à fonction nominale, donc, dans une subordonnée à fonction nominale ;

109 - 1.12, le subordonnant est l'adverbe when, à fonction adverbiale, donc, dans une subordonnée à fonction adverbiale.

110 Il en est tout autrement dans le cas des percontatives. Une même structure d'accueil (structure enchâssante) leur donne systématiquement le même statut syntaxique, statut nominal et non adverbial, même lorsque le subordonnant est un adverbe - where, when, why, how - ou la conjonction whether.

111 De cette remarque découle un test qui permet de vérifier le statut de la proposition en wh- comme problème de connaissance et comme percontative : elle admet alors la coordination de propositions introduites par des mots en wh appartenant à diverses catégories (coordination de problèmes de connaissances ayant tous un statut identique dans la structure d'accueil).

112 On peut mener ce test avec toutes les subordonnées nominales en wh-rencontrées dans le texte. On en essaiera 4, tirées des extraits 3 et 10 ci-dessus, et des extraits 22 et 23 cidessous

22. (1.37) Another day he asked her suddenly who Rosemary's best friends had been.

23. (1. 13-14) He behaved like a man who has had a shock, but to her question as to what was the matter, he replied briefly, 'Nothing.'

114 - 1. 14, extrait 23, what was the matter;

115 - 1.26, extrait 3, what was in his mind;

116 - 1.37, extrait 22, who Rosemary's best women friends had been;

$117-1.50$, extrait 10, what I'm trying to get at.

23 ' [...] to her question as to what was the matter and why, and whether she could help [...] (test positif: percontative)

3' She thought she saw what was in his mind and why, and whether she could help (test positif: percontative)

22' Another day, he asked her suddenly who Rosemary's best women friends had been and why, and since when and whether they had been very intimate. (test positif: percontative)

118 En revanche,

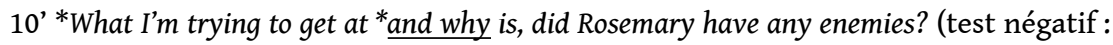
relative nominale / intégrative nominale / relative sans antécédent)

119 On a vu au $\S 7.1$ que, dans les percontatives, la relation inter-énonciative n'est pas nécessairement exploitée pour résoudre le problème de connaissance (ex. 3). Elle ne l'est que si le segment enchâssant le spécifie (ex. 22 et 23). On remarquera par ailleurs que l'enchâssement des percontatives ne garantit pas que l'on ait affaire à du discours rapporté : sa fonction est de signaler un problème de connaissance et de mettre en place une situation d'énonciation dérivée. La clé de l'indexation du problème de 
connaissance se trouve systématiquement dans l'enchâssante, qui pose l'existence de ce problème, mais également l'actant (she, dans 3), ou les actants concernés (référent de he comme siège du problème de connaissance, référent de her comme destinataire de la question, dans 22). L'indétermination des mots en wh- est toujours exploitée puisque c'est le mot en wh- qui est appelé à matérialiser le problème de connaissance à l'intérieur de l'enchâssée.

Dans l'extrait 10, 1. 50, en revanche, on a affaire à un dispositif de focalisation, une clivée en what. L'intégrative nominale ("relative » nominale) what I'm trying to get at présuppose I'm trying to get at something. Le déficit de spécification posé comme thème par what est élucidé dans le même énoncé (dans le focus qui vient immédiatement après is), et c'est précisément ce qui bloque la possibilité de coordination mise en évidence dans le test ci-dessus, et ce qui donne à la subordonnée nominale what I'm trying to get at son statut de relative (et non de percontative). Non seulement l'indétermination paradigmatique (caractéristique de wh-at) est résolue dans l'énoncé, mais c'est ce mécanisme qui justifie la structure de focalisation en what: elle permet de différer le contenu focalisé et de le présenter comme une élucidation. Le paradoxe, ici, c'est que l'élucidation référentielle de la relative nominale (what I'm trying to get at) est une question (did Rosemary have any enemies ?).

Les caractéristiques des percontatives sont donc les suivantes :

1/ elles ont un statut nominal qu'elles doivent à leur enchâssement dans une structure d'accueil ;

2/ en elles-mêmes, elles ne comportent aucun indice formel (pas d'inversion auxiliairesujet) permettant de les différencier des intégratives nominales («relatives sans antécédent ", comme what I'm trying to get at, 1.50, extrait 10) ou des intégratives adverbiales (circonstancielles, comme when she came in, 1. 12-13);

3 / elles sont systématiquement la représentation d'un problème de connaissance et relèvent à ce titre de la modalité épistémique ;

4/ c'est systématiquement dans la structure d'accueil, donc en dehors d'elles-mêmes, que se situe l'indice d'un problème de connaissance ;

126 5/ à la différence des questions, elles n'impliquent pas de situation inter-énonciative ni de sollicitation du co-énonciateur: seul le segment enchâssant peut spécifier l'un et l'autre.

\section{Questions et interrogation indirectes libres}

Le discours indirect libre se caractérise par son affranchissement - il n'est pas enchâssé dans une structure d'accueil. On adopte ici la distinction entre deux types de discours indirect libre (DIL) établie par Danon-Boileau 1982: DIL1, avec segment introducteur récupérable dans le contexte; DIL2, sans segment introducteur.

\subsection{Discours indirect libre 1}

24. (1.24) '[...] how things were going with her.'

25. (1. 24-25) 'Whether she was happy or unhappy.'

128 Ces deux contenus propositionnels sont dans du discours direct, mais ne sont pas des questions directes: il s'agit d'un premier type de discours indirect libre. Ils sont 
indexés en amont sur about what [?], ligne 23 : ce sont deux ré-instanciations de what, qui réfère au paradigme des thèmes pertinents dans la question directe about what [ ?]. Il $\mathrm{y}$ a en fait 4 ré-instanciations successives, donc 4 thèmes énumérés en extension (selon le modèle de la réponse de l'extrait $18,1.40$, examiné au $§ 6.2): 1 /$ herself; $2 /$ her friends ; 3 / how things were going with her; 4/ whether she was happy or unhappy. Cette réinstanciation fait qu'on se situe très près des caractéristiques des percontatives : pas d'inversion dans la structure de ces deux propositions (pas de modalité énonciative; pas de prédication, cf. $\$ 7.1$ ), et une énumération (asyndète) qui donne un même statut aux 4 segments de la ré-instanciation.

129 Mais le problème de connaissance n'est pas initié, il est simplement relayé par about what : le segment about what [?] est lui-même indexé sur la question de la ligne 21 (did Rosemary ever talk to you much [- about XYZ] ?).

130 Sans qu'il y ait enchâssement syntaxique, les deux propositions représentent deux problèmes de connaissance en raison de leur lien avec cette question. C'est la mémoire de la question posée ligne 21, réactivée et rethématisée ligne 23, qui permet de les interpréter comme tels, et de les assigner à la source énonciative de la ligne 21 (qui est aussi celle de 24-25), George. C'est ainsi que l'on peut trouver de l'indirect libre (de type 1) dans du discours direct. La source énonciative de la 1.21 met en place une situation dérivée d'énonciation dérivée. On a affaire à une double dérivation énonciative :

131 - dérivation 1: situation d'énonciation dans laquelle la source énonciative dérivée coïncide avec le personnage de George ; cf. he began to ask questions, 1. 19 ;

- dérivation 2: situation d'énonciation dans laquelle la source énonciative dérivée coïncide avec le personnage de Rosemary ; <Rosemary-talk to you much [+ about + wh- / réinstanciations]>.

En dehors de leur affranchissement syntaxique et de l'effort mémoriel qui seul permet de les interpréter, ils partagent les caractéristiques des percontatives, notamment - en dehors de l'absence d'inversion caractéristique des percontatives et de la dépendance contextuelle de leur interprétation épistémique - le fait de ne pas être soumis au coénonciateur pour élucidation. La question par laquelle l'énonciateur - siège de ces deux problèmes de connaissance - sollicite le co-énonciateur est celle de la 1.21 (cf. $§ 2.1$ et $\S$ 4), sur la validation et sur la quantification du contenu propositionnel <she-talk to you much>, dont dépend la résolution des problèmes de connaissance de 24 et de 25 .

134 A propos des mots en wh qui posent le thème de chacune de ces «interrogatives indirectes libres ", on remarque qu'ils réfèrent respectivement au paradigme adverbial de la caractérisation (how, modifieur de things were going on) et à un paradigme binaire (conjonction whether ouvrant l'alternative happy or unhappy).

135 Si l'on observe l'extrait suivant (26), on voit que sa syntaxe, à la différence de celle des extraits 24 et 25 , est celle des questions : cf. l'inversion had Rosemary et le point d'interrogation. La question s'interprète comme la représentation d'un problème de connaissance dont le thème est la quantification (how much; cf. note 14, § 2.2) des relations entre Rosemary et les Farradays.

26. (1. 57) How much had Rosemary seen of the Faradays?

Pour autant, il ne s'agit pas de discours direct: cf. l'absence de guillemets (qui établissent l'étanchéité du discours direct), et le prétérit perfect (had seen). Le prétérit perfect met en place un point de vue rétrospectif, qui s'interprète par rapport à une situation-repère. La situation-repère est celle posée à la ligne précédente (|...] he started 
asking about the Farradays.], et le point de vue, indexé sur cette situation-repère, est celui de George, via l'anaphorique he. L'interprétation du prétérit perfect est caractéristique du discours indirect. Le segment introducteur, qui représente une situation d'énonciation dérivée, comme dans le cas des extraits 24 et 25 , se trouve dans le contexte. Comme dans le cas de l'extrait 22 (percontative who Rosemary's best friends had been), c'est ce segment (et non l'interrogative indirecte libre) qui spécifie que le coénonciateur est sollicité : extrait 22 , he asked her $+C$; extrait 26 , he started asking about the Farradays.

\subsection{Discours indirect libre 2}

4. (1. 2) when had that begun?

27. (1. 2) What was the cause of it?

28. (1. 6-7) [...] when exactly had his abstraction become something more than natural?

A la différence du DIL1, il n'y a pas de segment introducteur identifiable dans le contexte comme mise en place d'une situation d'énonciation dérivée, et, comme dans le cas du DIL1, l'affranchissement qui caractérise le DIL2 ne permet pas de retrouver syntaxiquement le siège du problème de connaissance.

Les indices sont ailleurs et c'est le contexte large qui permet de retrouver la situation d'énonciation dérivée et d'identifier sa nature.

Ainsi les questions indirectes libres des lignes 2 (extraits 4 et 27) et 6-7 (extrait 28) sont indexées sur Iris :

140 - cf. 1. 1-2, Iris couldn't put it off any longer (dérivation 1);

141 - cf.1. 3, Iris could not put her finger definitely on the moment when it began (dérivation 1);

142 Les autres indices sont à la fois :

143 - des caractéristiques structurelles partagées avec le discours direct: inversion auxiliaire-sujet (ex. 4 et 28), sauf lorsque le sujet coïncide avec le thème en wh- (on peut hésiter à propos de l'ex. 27, qui admet deux interprétations syntaxiques - what attribut ou what sujet - puisque la question qui se pose autour de was est une question d'identification stricte et non d'attribution de propriété) ; le point d'interrogation ;

144 - des caractéristiques liées à l'emploi des temps, des aspects et des références personnelles partagées avec l'indirect classique. Ici, la dérivation énonciative s'opère par rapport au récit et ce sont les caractéristiques du récit qui sont importées, comme dans le discours indirect enchâssé. On distingue comme indices had begun (1. 2, ex 4) / had become (1.6) qui matérialisent un point de vue rétrospectif à partir de la situation repère - Iris couldn't put it off any longer / Iris could not put her finger definitely on the moment when it began -, situation de remémoration et de réflexion; his (1.6) qui implique un repérage de $3^{\mathrm{e}}$ personne (en rupture, autrement dit par rapport à un référent in absentia dans la situation repère).

Cette combinaison donne une représentation ${ }^{27}$ de discours intérieur, qui se distingue $\mathrm{du}$ récit par ses caractéristiques structurelles et par son intonation muette, et en osmose avec le récit par ses repérages. Malgré les caractéristiques structurelles partagées avec la question directe, la situation inter-énonciative par laquelle un co-énonciateur serait 
sollicité n'est pas pertinente et ne peut être activée : 1/ Iris est seule et 2/ il s'agit de discours intérieur.

Dans tous les cas (ex. 4, 27, 28), on a affaire à des prédications de problèmes de connaissance.

\section{Etudes de cas}

147 Les cas particuliers d'énoncés interrogatifs rencontrés dans ce corpus sont d'une part des segments interrogatifs indexés sur un énoncé en amont (\$ 9.1) et d’autre part des énoncés qui ont les caractéristiques syntaxiques d'énoncés déclaratifs, mais qui reçoivent un statut interrogatif (\$9.2).

\subsection{Segments interrogatifs indexés sur un énoncé en amont}

\subsubsection{Wh- in situ - demande de re-délimitation ou d'ajustement}

6. (1. 68-69) George said: 'What did Sandra Farraday think about it?"

'About what?'

29. (1. 23) 'Look here, Iris, did Rosemary ever talk to you much?'

Iris stared at him.

'About what?'

Syntaxiquement cette structure est sans ambiguïté : il n'y a que les questions (directes, directes libres, indirectes libres) qui permettent l'instanciation in situ, hors position initiale, à l'aide d'un paradigmatique en wh-. Ici, il s'agit de questions directes. Les deux occurrences de what - 1. 23, About what[ ?]; 1. 69 About what [?] - sont dans la rection de about. En anglais (à la différence du français), les questions comportant un mot en whin situ doivent être motivées en amont.

149 Ce qui distingue ce cas de figure, c'est précisément que l'instanciation à l'aide des mots en wh- (le pronom what, en l'occurrence) est une ré-instanciation, qui conserve toutes les données de l'énoncé qui précède, à l'exception de la détermination :

- 1.23 (ex. 29), [think] about $i t=>$ [ $\varnothing=$ think] About what?

- 1.69 (ex. 6), talk much $\varnothing \varnothing=>$ [talk] about what ( $\emptyset=>$ what).

Dans chaque cas, la question porte sur le thème de la question précédente : l'opinion de Sandra Farraday dans l'extrait 6, le thème prépondérant des confidences de Rosemary dans l'extrait 29 (cf. § 8.1).

Comme on l'avait constaté ci-dessus (\$ 6.2), la référence au paradigme pertinent, par ré-instanciation, assortie d'une indexation sur l'énoncé précédent, suffit à construire un énoncé viable: chacun de ces énoncés s'interprète comme un problème de connaissance (question directe épistémique) soumis au co-énonciateur avec frayage (référentiel) vers une nouvelle ré-instanciation en aval :

154 - extrait 6=> 'about her husband lending Rosemary pamphlets'

155 - extrait 29=> 'herself - her friends - how things were going with her. Whether she was happy or unhappy' (cf. § 8.1).

156 About what [ ?], 1. 23 comme 1.69, demande au co-énonciateur une nouvelle délimitation de sa question : les questions en wh- in situ exigent toujours un frayage en amont. Il ne s'agit pas uniquement d'une réappropriation du discours d'autrui, mais d'un recentrage 
destiné pragmatiquement à sonder le co-énonciateur (cf. Iris stared at him, l. 22), et à éviter de se livrer de façon trop large.

A la ligne 69, la ré-instanciation de la case structurelle du complément de about réintroduit l'indétermination et une référence qualitative au paradigme des référents possibles de it: autrement dit cette question demande une élucidation de it. Elle fonctionne presque comme une question-écho.

8. (1. 50-51) 'What I'm trying to get at is, did Rosemary have any enemies?'

'Amongst other women?'

La question directe amongst other women[?] (1.51, ex. 8) s'apparente à la question about what [?] (1.23, ex. 29) : elle soumet au co-énonciateur une nouvelle délimitation de sa question (1.50). La différence est que cette délimitation est suggérée à la ligne 51, alors qu'elle était demandée à la ligne 23. Cette suggestion s'apparente ainsi aux cas de figure examinés au $§ 9.2$.

\subsubsection{Echo partiel}

7. (1. 48-49) 'Did Rosemary ever say she was afraid of anybody?'

'Afraid ?' Iris stared.

Afraid[ ?] est un écho partiel de la ligne précédente. Il s'agit d'une question directe, assortie d'une intonation creusée, qui porte sur la pertinence de afraid. Comme dans le cas des questions comportant un mot en wh in situ (§ 9.1.1), il y a nécessairement un frayage en amont.

La motivation de cette question pourrait être la remise en cause de la pertinence de afraid. Mais, ici, le contexte en amont - George's response to it surprised her (1. 46-47) rend plus vraisemblable que le personnage d'Iris (source énonciative) attende une élucidation de la part du co-énonciateur. La situation inter-énonciative est exploitée pour demander une explicitation de la pertinence de afraid (modalité épistémique) mais également pour sonder le co-énonciateur et mettre au jour la motivation de la question de la ligne 48 .

\subsubsection{Question tags}

9. (1. 84) 'Knocked about a good deal, hasn't he?'

30. (94-95) 'Rosemary saw rather a lot of Anthony Browne, didn't she?'

31. (109-110) Such a gay party it had been, or hadn't it?

Les question tags ${ }^{28}$ sont eux aussi systématiquement indexés sur un énoncé en amont, énoncés de discours direct dans les extraits 9 et 30, énoncé de discours indirect libre dans l'extrait 31.

Les caractéristiques de ces trois tags sont formellement les mêmes: ils sont interronégatifs et indexés sur un énoncé de type assertif à polarité positive.

Dans l'extrait 9, l'énonciateur donne comme validé un contenu propositionnel ( $\mathrm{p}:<\varnothing$ knock about a good deal>. Les deux éléments effacés (sujet et élément nodal have) sont rétablis dans le tag. L'ellipse (anaphore mémorielle) active le lien avec l'antécédent du sujet $\varnothing$ et produit une assertion moins dépendante de l'énonciateur, difficilement contestable. Le question tag est une question biaisée (rhétorique), qui sert avant tout à ouvrir la fenêtre inter-énonciative caractéristique de la question directe, avec sollicitation du co-énonciateur ${ }^{29}$. Ce qui est proposé à la question, c'est p', ce qui revient à une remise en question de p' (non-validation de la relation prédicative) dans une 
situation inter-énonciative sollicitant la confirmation du co-énonciateur. C'est effectivement cette confirmation qui vient à la ligne suivante (1. 86) : 'He's travelled a lot, of course.'

Dans l'extrait 30, on a également affaire à un question tag rhétorique. C'est le retour à $\mathrm{p}$ (validation de <Rosemary- see rather a lot of Anthony Browne $>$ ) qui est attendu et le détour par p' (didn't she[ ?]) se présente comme une remise en cause de p' (non-validation p' mise en question). Il s'agit d'exploiter la caractéristique centrale de la question l'ouverture d'une situation inter-énonciative en vue de solliciter le co-énonciateur pour demander au co-énonciateur son adhésion à l'assertion. La confirmation vient à la ligne suivante (1.96) : 'Yes - yes, she did.'

Le contexte de l'extrait 31 est un contexte de discours indirect libre, autrement dit de discours intérieur, dont le siège, et la source énonciative, est Iris. Il s'agit d'un point de vue rétrospectif (analeptique had been) sur l'anniversaire de Rosemary. Such a gay party it had been est une assertion exclamative et/ou une exclamation assertive. La relation <itbe a gay party> est donnée comme validée (p). Une différence d'importance par rapport aux extraits 9 et 30 tient à la présence de or, qui pose une alternative. Pour cette raison, il s'agit d'une véritable remise en cause, que l'on peut gloser de la façon suivante : ou bien est-ce p' (la non-validation de <it-be such a gay party>) qui a été le cas ? Comme il s'agit de discours intérieur, cette question représente un doute, et les polarités antagonistes de l'énoncé représentent un débat intérieur.

\subsection{Structure déclarative à modalité interrogative}

32. (1. 51-53) 'Amongst other women ?'

'No, no, not that kind of thing. Real enemies. There wasn't anyone - that you

knew of - who - who might have had it in for her?'

33. (1.94-98) 'Rosemary saw rather a lot of Anthony Browne, didn't she?'

'Yes - yes, she did.'

'But she hadn't known him very long - he was more or less of a casual acquaintance?'

Les deux énoncés 'There wasn't anyone - that you knew of - who - who might have had it in for her?' (1.52-53) et 'But she hadn't known him very long - he was more or less of a casual acquaintance?' (1.97-98) ont un point commun : ils ont une syntaxe déclarative et un statut illocutoire interrogatif. Le statut illocutoire est marqué par le point d'interrogation et en conséquence par le contour intonatif.

Dans l'extrait 32, on a affaire à une question-éclaireur (tentative question) qui soumet au co-énonciateur une représentation de la prédication d'existence construite sur une conjecture (there might have been someone who might have had it in for her) et qui pourrait se formuler sur le modèle de la question de la ligne 33 (ex. 13) : do you think there was someone who might have had it in for her? Les deux relatives ont une fonction référentielle restrictive et il n'y a qu'une prédication, prédication d'existence d'un humain muni des deux propriétés données dans les deux relatives.

La stratégie énonciative consiste à représenter p' (contenu propositionnel négatif there wasn't anyone...) et à lui associer une modalité interrogative. Autrement dit, l'énonciateur soumet p' au co-énonciateur dans le cadre de la situation interénonciative mise en place par la modalité interrogative. Et il sollicite le co-énonciateur pour traiter p'. Pourquoi ne pas utiliser $\mathrm{p}$ (There was someone...)? Soit l'énoncé contiendrait une question tag, (there was someone [...], wasn't there ?) et on retrouverait le 
modèle rencontré ci-dessus (ex. 9 et 30, § 9.1.3), à savoir une assertion à propos de laquelle l'énonciateur solliciterait le co-énonciateur pour corroboration. Soit l'énoncé proposerait $\mathrm{p}$ ? (There was someone who [might have] had it in for her?) et il serait soustendu par un présupposé d'existence. Or rien dans le contexte ne permet d'étayer à ce stade cette présupposition et de lui donner un statut de conjecture conclusive, ou de quasi-certitude. Il reste à soumettre p' au co-énonciateur et à le solliciter pour confirmer ou pour infirmer p' (et dans ce cas établir p).

L'extrait 33 (1. 97-98), 'But she hadn't known him very long - he was more or less of a casual acquaintance?' est théoriquement ambigu : soit les deux contenus propositionnels sont concernés par la modalité interrogative, soit, plus vraisemblablement, uniquement le second. Et ici, c'est $\mathrm{p}$ qui est soumis au co-énonciateur : on en revient à l'analyse du précédent paragraphe. Il s'agit d'une conjecture conclusive qui s'appuie sur she hadn't known him very long, notamment. L'énonciateur soumet sa conjecture, ou sa conviction ( $\mathrm{p}$ : he was more or less of a casual acquaintance) au co-énonciateur pour traitement, à l'égal d'un problème de connaissance, mais avec attente de confirmation: Cette question se présente comme une question biaisée.

\section{Conclusion}

170 On a défini l'interrogation d'une part comme un statut illocutoire, d'autre part comme une modalité énonciative, et l'énoncé interrogatif comme une prédication munie de cette modalité et de ce statut illocutoire. On a envisagé la modalité comme un phénomène à la fois sémantique - une détermination du sens construit d'un énoncé - et pragmatique - d'ajustement à une situation inter-énonciative. La force illocutoire mobilise les deux pour faire aboutir une intention d'obtenir.

Porteur de modalité et de statut illocutoire, l'énoncé interrogatif est motivé en amont (évaluation de données situationnelles ou contextuelles) et en aval (visée énonciative, ajustement inter-énonciatif). La plurimodalité inhérente à la question peut se comprendre comme une chaine modale - d'évaluation de données situationnelles ou contextuelles, d'identification d'un problème de connaissance, de nécessité d'élucidation, de conjecture, de sollicitation inter-énonciative.

La question canonique se structure de telle sorte que soit placé en position initiale le thème de l'incertitude. Les implications pragmatiques de l'interrogation - et donc l'exploitation de la situation inter-énonciative - varient en fonction de son statut discursif :

173 L'incertitude est d'ordinaire soumise à un co-énonciateur pour résolution dans les questions directes canoniques, qui sont des prédications. La situation inter-énonciative est exploitée pour solliciter le co-énonciateur, soit pour le sonder, soit pour en obtenir l'élucidation d'un problème de connaissance, la reconnaissance d'une assertion, la confirmation ou l'infirmation d'un contenu propositionnel; la sollicitation interénonciative peut aller jusqu'à l'injonction.

Les percontatives ( interrogatives indirectes ») se définissent par leur enchâssement et elles reçoivent leur statut de la structure d'accueil qui seule permet de les identifier. La structure d'accueil met en place une situation d'énonciation dérivée et c'est elle qui signale systématiquement la percontative; ce contenu représente dans l'immense 
majorité des cas une problème de connaissance, qui n'est pas a priori soumis à un coénonciateur pour élucidation, sauf si le segment introducteur le spécifie.

Les interrogatives indirectes libres ont des caractéristiques qui les rapprochent soit des percontatives, soit des questions. Elles ne sont pas enchâssées, mais elles peuvent se trouver à proximité d'un segment introducteur (DIL1). Les percontatives indirectes libres ont alors des caractéristiques (repérages temporels, aspectuels, personnels) identiques à celles des percontatives. Elles peuvent également, sans segment introducteur (DIL2), concentrer les caractéristiques structurelles des questions directes, et les repérages propres au discours indirect. Les questions indirectes libres ne sont pas a priori destinées à solliciter un co-énonciateur dans le cadre d'une situation inter-énonciative : ces questions relèvent du discours intérieur.

La sollicitation inter-énonciative est l'invariant de la question directe, qu'elle soit question rhétorique (cf. les études de cas des $\$$ 9.1.3 et $§ 9.2$ ) ou question canonique.

\section{BIBLIOGRAPHIE}

chabert, E. (2016). 'Disentangling free relatives, indirect interrogative and exclamative subordinate clauses', Communication aux journées de l'ALAES, accessible en ligne : https:// alaesfrance.files.wordpress.com/2016/03/e-chabert.pdf.

culioli, A. (1990). Pour une linguistique de l'énonciation, tome 1. Paris / Gap : Ophrys.

Damourette, J. \& Pichon E. (1911-1936) [1970], Des mots à la pensée. Essai de grammaire de la langue française, tome V. Paris : Editions d'Artrey.

Danon-Boileau, L. (1982). Produire le fictif. Paris : Klincksieck.

Danon-Boileau, L. \& Morel M.-A. (2003). « Le locuteur vicariant », in Le Sujet (dir. Jean-Marie Merle), Bibliothèque de Faits de Langues. Paris / Gap : Ophrys, 235-246.

Delveroudi, R. (2004). «Phrases interrogatives à valeur injonctive en grec et en français », in Contrastes (dir. Lucie Gournay \& Jean-Marie Merle), Paris / Gap : Ophrys, 141-154.

Gaffiot, F. (1992) [1934], Dictionnaire latin-français. Paris : Hachette.

Groussier, M.-L. \& Rivière C. (1996). Les Mots de la linguistique. Paris / Gap : Ophrys.

Guillaume, B. (2006). Approche énonciative des question tags en anglais, Cahiers de recherche, numéro spécial (collection dirigée par Janine Bouscaren). Paris / Gap : Ophrys.

Le Goffic, P. (2005). « Ce qui, ce que : CQFD ». Pratiques, 125-126, 25-47. Villeneuve d'Ascq : Presses universitaires du Septentrion. https://www.persee.fr/doc/ prati_0338-2389_2005_num_125_1_2059

Lefeuvre, F. (2015). « Analyse grammaticale et sur corpus de l'expression c'est quoi ( ?) », in La dia-variation en français actuel. Études sur corpus, approches croisées et ouvrages de référence. Dostie G. et Hadermann P. (dir.), Peter Lang, coll. Sciences pour la communication, 39-62. https:// halshs.archives-ouvertes.fr/halshs-01519591/document 
Mélis, G. (2006). « Peut-on différencier l'opération de parcours ? », in Le Parcours, concept métalinguistique de la TOE (dir. Lucie Gournay \& Gérard Mélis). Corela, 4 http:// journals.openedition.org/corela/1401; DOI : 10.4000/corela.1401.

Merle, J.-M. (2002). « Want », in Linguistique contrastive et traduction (dir. Jacqueline GuilleminFlescher), tome 6. Paris / Gap : Ophrys : 151-181. http://hal.archives-ouvertes.fr/hal-00378859

Merle, J-M. (2006). «Wh- et la référence qualitative aux paradigmes », in Le Qualitatif (dir. Jean-Claude souesme), Cycnos, vol. 23, n 1. Université de Nice, 25-43. http://revel.unice.fr/ cycnos/index.html?id=294.

Merle, J.-M. (2009). Présentation générale de La prédication, collection Faits de Langues, $\mathrm{n}^{\circ}$ 31-32 (dir. J.-M. Merle). Paris : Ophrys, 5-12. http://hal.archives-ouvertes.fr/hal-00374896.

Merle, J.-M. (2017). « La prédication - approche de la théorie des opérations prédicatives et énonciatives ", Corela, volume Hors-Série 22 (dir. Laurence Vincent-Durroux), mis en ligne le 05 novembre 2017, URL : http://journals.openedition.org/corela/4959 ; DOI : 10.4000/corela.4959

wyld, н. (2001). Subordination et énonciation, Cahiers de Recherche, numéro spécial, (collection dirigée par Janine Bouscaren). Paris / Gap : Ophrys.

\section{NOTES}

1. Les termes employés par grammairiens et linguistes viennent tous étymologiquement de verbes latins, qui contiennent déjà l'orientation sémantique exploitée ici.

- Rogo, as, are, avi, atum

1. Interroger, questionner [Cic.] 1.1. + interrogative indirecte ; 1.2. + aliquem ; 1.3. aliquid //// 2.1. rogare aliquem sententiam demander à qn son avis; 2.2. rogare populum consulter le peuple sur une loi (faire une proposition de loi) [Cic.] ; 2.3. rogare magistratum populum [Liv.] demander au peuple [qu'il désigne] un magistrat ; 2.4. rogare milites sacramento [Caes.] (consulter les soldats selon une formule de serment) faire prêter serment, enrôler /// 3. chercher à obtenir en priant, prier, solliciter, faire une requête (aliquid ab aliquo / aliquem de aliqua re) ; rogare ut / ne (+ contenu propositionnel au subj.) [Cic. Caes.] ; rogare + infinitive [Catul.] (Gaffiot 1992 [1934])

- Interrogo (inter + rogo), as, are, avi, atum

1. Interroger, questionner (1.1. + aliquem / 1.2.+ interrogative indirecte + 1.3. + aliquid [sur qch] 1.4. + aliquem aliquam rem [Cic] + sententias demander un avis // 1.5. interrogare aut interrogari [Cic.]) /// 2. Poursuivre en justice (aliquem lege : qqn selon une loi [Cic.] (Gaffiot 1992 [1934])

- Derogo

1. Abroger une ou plusieurs dispositions d'une loi ; déroger à une loi [Cic.] (Derogari ex hac lege);

2. Oter, retrancher ([Cic.] aliquid de honestate déroger à ce qu'exige l'honneur; aliquid ex aequitate [Cic.] porter atteinte à l'équité. (Gaffiot 1992 [1934])

2. - Quaero, is, ere, quaesivi, situm

1. Chercher (qn, qch) [Cic. Caes.]; 2. Chercher en vain, rechercher, réclamer [Cic. Sall.]; 3. Chercher à obtenir, se procurer [Cic.] ; 4 . Rechercher, mettre en question ; 5 . Chercher à savoir, demander ; chercher à savoir en justice, faire une enquête, instruire $=>$ de servo in dominum [Cic.] mettre un esclave à la question, concernant son maître. (Gaffiot 1992 [1934])

- Quaestio, onis f. 1. Recherche ; 2 . Interrogatoire ; 3 . question, enquête ; problème, thème de discussion ; 4. Enquête judiciaire ; information ; 5. Question, torture. (Gaffiot 1992 [1934])

3. Terme emprunté à Damourette \& Pichon (1911-1936), § 1412-1430, repris par Le Goffic (2005), Lefeuvre (2015), Merle (2006).

4. Cf. Merle (2017). 
5. Le trait d'union (-) représente ici la ligature qui unit le sujet et le prédicat, selon une interprétation néo-aristotélicienne de la prédication, vue comme l'assemblage d'un apport (prédicatif) et d'un support (subjectal), assemblage muni de ses déterminations, nominales et verbales, et d'une détermination modale qui lui donne le statut de relation énonciative et de prédication (cf. Merle 2009, 2017). La barre oblique (/), quant à elle, représente une alternative : elle représente une opération sur le paradigme (ex. Did Rosemary / Mr Faraday ever talk to you much ? < => Did Rosemary or Mr Faraday ever talk to you much ?). Sujet et prédicat ne constituent pas une alternative, alors que tous les éléments susceptibles d'instancier une place structurale donnée constituent un paradigme (que l'on peut considérer comme un paradigme d'alternatives). La ligature (-) opère dans la chaîne linéaire et intervient alors que les choix paradigmatiques (/) sont effectués et les cases structurales instanciées pour produire un énoncé donné : c'est le ligateur $r$ de $a r b$, autrement dit le nœud prédicationnel. La ligature est prototypiquement représentée (marqueur) et/ou opérée (opérateur) par le premier élément verbal, la fonction verbale étant nodale, modale, prédicative (cf. Merle 2006). La question canonique directe s'annonce par la position du ligateur, position initiale dans les questions binaires ( $\mathrm{rab}$ ), immédiatement après le mot en wh- dans les questions paradigmatisantes (wh-rab; ou wh-rb quand le mot en whest sujet).

6. Ce qui correspond à l'interprétation épistémique de la question (contenu de sens présenté comme problème de connaissance).

7. Ce qui correspond à différents cas d'exploitation pragmatique de la situation inter-énonciative (l'énonciateur sonde ou met à l'épreuve le co-énonciateur, par exemple).

8. I signifie Intérieur (zone de validation) ; E signifie Extérieur (zone de non-validation). Il s'agit de deux zones complémentaires du domaine notionnel, correspondant respectivement à la validation (localisation dans l'intérieur I : Rosemary had enemies) d'un contenu propositionnel, que l'on appelle par convention $\mathrm{p}$, et à la non-validation (localisation dans l'extérieur $\mathrm{E}$ : Rosemary didn't have any enemies) de ce contenu propositionnel, que l'on appelle p'. Validation (p) et nonvalidation ( $p$ ') sont susceptibles d'être assertés. Dans la question binaire canonique (did Rosemary have any enemies ?), l'équipondération (représentée $p, p$ ') dont parle Culioli correspond au fait que ni p (en I), ni p' (en E) n'est sélectionné, et que l'énoncé interrogatif direct canonique donne un poids égal aux deux pour exprimer une incertitude absolue.

9. Culioli poursuit ainsi : «Il a recours à autrui pour rompre le parcours [...]».

Le concept de "parcours" n'est pas utilisé ici, car cognitivement peu rentable et en contradiction avec la possibilité linguistique de représenter directement en énoncé l'incertitude (qui relève de la modalité) ou l'indifférenciation (qui relève de la détermination), autrement dit l'instabilité référentielle (concernant un état de fait, un événement, un référent), et d'établir directement en énoncé une référence qualitative aux paradigmes. Cf. Mélis (2006), Merle (2006).

10. Did n'est pas concerné par l'agentivité du sujet, mais par la validation / non-validation de la relation prédicative qui se noue autour de lui : il est en cela porteur de sens (c'est pour cette raison qu'il n'est pas 'dummy').

11. Cf. Merle (2006) : http://revel.unice.fr/cycnos/index.html?id=294.

12. Structurel et structural concernent l'un et l'autre la structure. Structurel s'applique à l'organisation, au fonctionnement de la structure ; structural s'applique à la place ou à la fonction d'un élément à l'intérieur de la structure.

13. Instanciation : "Opération consistant à remplir au moyen de notions spécifiques les places d'un schéma de lexis. L'instanciation est une opération de choix notionnel.» (Groussier \& Rivière, 1996 : 106)

En tenant compte de l'adéquation entre le schéma de lexis et sa structuration syntaxique, on peut ajouter que l'instanciation est nécessairement maintenue quand le schéma de lexis se structure ; l'instanciation est aussi l'occupation d'une case structurale identifiable dans la chaine linéaire. La 
question directe en wh- est une instanciation qui invite le co-énonciateur à une ré-instanciation selon un paradigme donné, en adéquation avec la place instanciée par wh- (cf. Merle 2006).

14. Cf. Merle (2006 : 29) : « En anglais, aucun des paradigmes [auxquels renvoient les mots en wh-) du système n'est spécialisé dans le quantitatif: dans tous les cas, chacun des paradigmes est défini de façon qualitative exclusivement. Force est donc de recourir au qualitatif pour signifier le quantitatif [quantité, nombre, degré] : cf. how much, how many, how fast, how tall, how small, etc., littéralement, "beaucoup comment", «nombreux comment", « rapide comment», "grand comment ", « petit comment », etc. »

15. Pour cette raison, Groussier \& Rivière (1996: 120-121) placent l'interrogation comme l'assertion dans les «modalités du 1er ordre»: «Les modalités du $1^{\text {er }}$ ordre ou modalités fondamentales sont celles qui constituent, de la part de l'énonciateur, le choix d'un plan modal : plan de la conformité à ce que l'énonciateur considère comme un fait pour l'assertion (affirmative et négative) et l'interrogation, [...]».

Les modalités du $1^{\mathrm{er}}$ ordre sont les modalités de la connaissance. Demander did Rosemary have any enemies[?] revient 1/ à évaluer des données situationnelles ou contextuelles (Rosemary est morte assassinée) ; 2/ à structurer [relation prédicative] un schéma de lexis pertinent (Rosemary-haveenemies) ; 3 / à ne lui donner ni le statut de relation validée, ni celui de relation non-validée, soit parce qu'on n'a pas - ou pas assez - d'indices pour corroborer ou pour invalider cette intuition (George découvre des pans insoupçonnés de la vie de Rosemary), soit pour sonder le coénonciateur ; 4/ à ouvrir un espace d'interlocution (rôle de la question directe canonique) pour soumettre un problème de connaissance au co-énonciateur (support référentiel : Iris) ; 5/ dans la question canonique (problème de connaissance soumis au co-énonciateur), le but de l'énoncé est de déclencher une réponse.

16. Les questions dites rhétoriques exploitent la fonction illocutoire de la question, dont le rôle est d'ouvrir explicitement et de maintenir ouverte une fenêtre inter-énonciative sollicitant le coénonciateur (force illocutoire de la question) pour lui faire admettre un contenu de sens, présenté sous la forme d'une question biaisée (assertion déguisée en question : plurimodalité illocutoire). La différence entre l'assertion (she had many enemies) et la question rhétorique (didn't she have many enemies? [l'interprétation rhétorique, comme l'interprétation épistémique, dépend du contexte]) est dans le fait que l'assertion est prise en charge par la source énonciative, alors que la question rhétorique (comme assertion déguisée) sollicite l'adhésion du co-énonciateur.

C'est pour cette raison que les méta-termes d'interrogation directe, de question directe, de demande sont pertinents pour décrire ce phénomène pragmatique et énonciatif, quelle qu'en soit la motivation. Ils impliquent une sollicitation: consultation et interrelation (interrogation, cf. introduction note 1); recherche, élucidation, pression pour obtenir [une réponse, une coopération, un comportement] (question, cf. introduction note 2) ; relation par laquelle on confie ou impose une mission [la demande de réponse, la demande d'attention en font partie, mais également divers types de directives] (demande, $\mathrm{cf}$. lat. mandare = confier; lat. demandare : donner mandat à , missionner pour).

C'est pour cette raison aussi que le potentiel de plurimodalité illocutoire de la question déborde la possibilité de réaliser une assertion sous forme de question (rhétorique) ; la question peut aussi être détournée pour énoncer une injonction (Trace called out to her, 'Will you close the door behind you? [Turner]') (cf. Delveroudi 2004), ou encore pour donner à un énoncé exclamatif les caractéristiques structurelles de la question ('Oy, was I thirsty! Oy, was I thirsty! [Brenner]).

17. Cette remarque s'appuie sur la complémentarité du certain (assertable) et du non-certain (non assertable, mais qui peut être représenté linguistiquement sous différentes formes de détermination épistémique). Il s'agit de la modalité (modus, comme détermination énonciative) et non du contenu propositionnel (dictum) dont la validation ( $\mathrm{p}$ ) ou la non validation ( $\mathrm{p}$ ') est donnée comme certaine ( $\mathrm{p} / \mathrm{p}^{\prime}$ : $\mathrm{p}$ est le cas / p' est le cas, c'est-à-dire $\mathrm{p}$ n'est pas le cas, et éventuellement autre que $\mathrm{p}$ est le cas) ou incertaine ( $\left.\mathrm{p}, \mathrm{p}^{\prime}\right)$. Par ailleurs donner comme certain ou comme non 
certain un contenu énonciatif ne préjuge ni de l'objectivité d'un énoncé, ni de son caractère véridique (modalité aléthique). Le mensonge et l'ironie exploitent la modalité du certain et du non-certain respectivement pour dissimuler la vérité ou, en aval, égarer le co-énonciateur (le destinataire de l'énoncé), et pour mener par un détour à la vérité que l'énonciateur cherche à faire admettre, en aval, au co-énonciateur.

18. Le certain correspond à I, l'intérieur du domaine notionnel, et il constitue l'un des pôles du gradient épistémique. Le non-certain correspond à E, l'extérieur du domaine notionnel. L'incertain absolu [don't know] est l'autre pôle de ce gradient. Certain et incertain absolu sont deux centres organisateurs de la modalité de la connaissance. La question canonique correspond au pôle incertain absolu.

19. Cf. Merle $(2002,2017)$.

20. Cf. Wyld (2001).

21. Enonciateur et co-énonciateur sont deux coordonnées énonciatives. La négociation (pragmatique) de l'allocution ou de l'interlocution active les rôles pragmatiques de locuteur (source) et d'allocutaire (destinataire) ou de colocuteur (destinataire appelé à prendre son tour de parole). Dans un contexte de question canonique, le destinataire est appelé à être colocuteur (le problème de connaissance lui est soumis pour élucidation). Cf. Danon-Boileau \& Morel (2003).

22. Zone $\mathbf{E}$ de l'extérieur : cf. $\S 3$ et notes 8, 17, 18.

23. Cf. Wyld (2001) à propos de la problématique générale des situations d'énonciation dérivées.

24. Cf. Merle (2017: § 4).

25. Le terme de percontative, inventé par Damourette \& Pichon, vient d'un verbe déponent latin verbe de voix moyenne - qui se différencie en cela des verbes rogare, interrogare (cf. note 1), quaerere (cf. note 2) ou demandare (cf. note 16), qui tous sont transitifs et représentent une sollicitation inter-énonciative. Percontative en raison de son étymologie déponente (qui correspond aux pronominaux de voix moyenne en français), dit bien que le co-énonciateur n'est pas nécessairement sollicité (cf. se demander).

Percontor, aris, ari, atus sum 1. S'enquérir, interroger, questionner; s'informer, se demander (aliquem aliquid / ab aliquo / ex aliquo (Gaffiot 1992 [1934])

26. Pour un examen complet de la distinction entre relative nominale et interrogative nominale, voir Chabert (2016). Voir également Leonarduzzi (2004) pour une présentation plus générale de la subordination percontative.

27. Il ne s'agit pas de discours rapporté, mais de discours représenté.

28. Voir Guillaume (2006) pour une étude complète des question tags.

29. Cet énoncé peut s'envisager comme une assertion avec accusé de réception.

\section{RÉSUMÉS}

Cet article porte sur les caractéristiques de la question et de l'interrogation. Dans une perspective énonciative, il présente les différents types de structuration, et leurs implications énonciatives et pragmatiques - modales et illocutoires. Il pose la question de la modalité épistémique et de sa pertinence, et de la plurimodalité sous-jacente à la motivation des questions et de l'interrogation. Il observe de quelle façon se construit la force illocutoire d'une question, et de quelle façon elle se neutralise dans les percontatives (interrogatives indirectes). Il met en évidence la relation interénonciative et de quelle façon elle est exploitée pour solliciter le destinataire (co-énonciateur). 
This paper sets out to examine questions and interrogation from an enunciative perspective: the various types of structuration are presented in connection with their enunciative and pragmatic - modal and illocutionary - implications. It examines the relevance of epistemic modality, and explores the underlying plurimodality which motivates questions and interrogation, and in what way illocutionary force builds up, and can be neutralised in percontative clauses (interrogative content clauses). It sheds light on the inter-enunciative relation and the way it is exploited.

\section{INDEX}

Mots-clés : question, percontatives, relation inter-énonciative, plurimodalité, statut illocutoire Keywords : questions, percontative clauses, inter-enunciative relation, plurimodality, illocutionary force

\section{AUTEUR}

\section{JEAN-MARIE MERLE}

BCL (Bases, Corpus, Langages), UMR 7320, Université Nice Sophia Antipolis jean-marie.merle@unice.fr 